\title{
BMJ Global Health Effects of nutrition interventions during pregnancy on low birth weight: an overview of systematic reviews
}

Katharina da Silva Lopes, ${ }^{1}$ Erika Ota, ${ }^{1}$ Prakash Shakya, ${ }^{2}$ Amarjargal Dagvadorj, ${ }^{3}$ Olukunmi Omobolanle Balogun, ${ }^{3}$ Juan Pablo Peña-Rosas, ${ }^{4}$ Luz Maria De-Regil, ${ }^{5}$ Rintaro Mori ${ }^{3}$

To cite: da Silva Lopes K, Ota E, Shakya P, et al. Effects of nutrition interventions during pregnancy on low birth weight: an overview of systematic reviews. BMJ Glob Health 2017;2:e000389. doi:10.1136/ bmjgh-2017-000389

Handling editor Prashant Jha

- Additional material is published online only. To view please visit the journal online (http://dx.doi.org/10.1136/ bmjgh-2017-000389).

Received 1 May 2017 Revised 15 August 2017 Accepted 16 August 2017

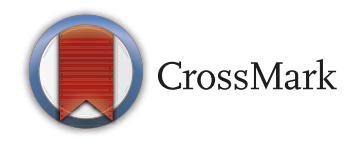

${ }^{1}$ Global Health Nursing, Graduate School of Nursing Science, St. Luke's International University, Tokyo, Japan ${ }^{2}$ Faculty of Social Sciences, Kyorin University, Tokyo, Japan ${ }^{3}$ Department of Health Policy, National Center for Child Health and Development, Tokyo, Japan ${ }^{4}$ Evidence and Programme Guidance, Department of Nutrition for Health and Development, World Health Organization (WHO), Geneva, Switzerland

${ }^{5}$ Nutrition International, Research and Evaluation, Ottawa, Canada

Correspondence to Professor Erika Ota; ota@sicn.ac.jp

\section{ABSTRACT}

Introduction Low birth weight (LBW, birth weight less than $2500 \mathrm{~g}$ ) is associated with infant mortality and childhood morbidity. Poor maternal nutritional status is one of several contributing factors to LBW. We systematically reviewed the evidence for nutrition-specific (addressing the immediate determinants of nutrition) and nutrition-sensitive (addressing the underlying cause of undernutrition) interventions to reduce the risk of LBW and/or its components: preterm birth (PTB) and small-forgestational age (SGA).

Methods We conducted a comprehensive literature search in MEDLINE, EMBASE, CINAHL and the Cochrane Database of Systematic Reviews (September 2015). Systematic reviews of randomised controlled trials focusing on nutritional interventions before and during pregnancy to reduce LBW and its components were eligible for inclusion into the overview review. We assessed the methodological quality of the included reviews using $A$ Measurement Tool to Assess Reviews (AMSTAR), PROSPERO: CRD42015024814.

Results We included 23 systematic reviews which comprised 34 comparisons. Sixteen reviews were of high methodological quality, six of moderate and only one review of low quality. Six interventions were associated with a decreased risk of LBW: oral supplementation with (1) vitamin A, (2) low-dose calcium, (3) zinc, (4) multiple micronutrients (MMN), nutritional education and provision of preventive antimalarials. MMN and balanced protein/energy supplementation had a positive effect on SGA, while high protein supplementation increased the risk of SGA. High-dose calcium, zinc or long-chain n-3 fatty acid supplementation and nutritional education decreased the risk of PTB.

Conclusion Improving women's nutritional status positively affected LBW, SGA and PTB. Based on current evidence, especially MMN supplementation and preventive antimalarial drugs during pregnancy may be considered for policy and practice. However, for most interventions evidence was derived from a small number of trials and/or participants. There is a need to further explore the evidence of nutritionspecific and nutrition-sensitive interventions in order to reach the WHO's goal of a $30 \%$ reduction in the global rate of LBW by 2025.

\section{Key questions}

What is already known about this topic?

- Low birth weight (LBW, newborns weighing less than $2500 \mathrm{~g}$ at birth) is a significant global health problem and a major underlying cause of perinatal mortality and morbidity. LBW babies have an increased risk of non-communicable diseases in adult life which consequently add to the global burden of disease.

- While several factors contribute to LBW, to a great extent, it is affected by poor maternal nutrition and health.

\section{What are the new findings?}

- This overview of systematic reviews summarised available effective nutrition-specific and nutritionsensitive interventions during pregnancy for the outcome of LBW.

- Oral supplementation with vitamin $A$, low-dose calcium, zinc, multiple micronutrient (MMN) supplements, nutritional education and preventive antimalarials decreased the risk of LBW.

\section{Recommendations for policy}

- The identified interventions will provide the basis to formulate recommendations and to develop a guideline to tackle the third global nutrition target 2025: a 30\% reduction in LBW. Based on current evidence, especially MMN supplementation and preventive antimalarial drugs during pregnancy may be considered for policy and practice.

\section{INTRODUCTION}

Low birth weight (LBW) is a major public health problem. Globally, approximately $16 \%$ of infants are born weighing less than $2500 \mathrm{~g}$, which represents more than 22 million LBW babies per year. ${ }^{1}$ Over $95 \%$ of these infants are born in low-income and middle-income countries. In South Asia, about one quarter (28\%) of all infants are born with LBW. In sub-Saharan Africa and in Latin America/ Caribbean, the LBW rates are estimated to be 
$13 \%$ and $9 \%$, respectively. ${ }^{1}$ There is a high probability that the incidence of LBW is underestimated as almost $50 \%$ of all newborns are not weighed at birth. ${ }^{1}$

The WHO defines LBW as weight at birth less than $2500 \mathrm{~g}$ irrespective of the gestational age of the infant. ${ }^{23}$ Based on epidemiological observations, infant mortality rate rapidly rises for newborns weighing less than $2500 \mathrm{~g}$ at birth. ${ }^{3}$ LBW includes very low birth weight (VLBW; less than $1500 \mathrm{~g}$ ) and extremely low birth weight (ELBW; less than $1000 \mathrm{~g}$ ) infants, who have the highest risk of adverse outcomes. $^{24}$

LBW is a complex syndrome that arises through preterm birth (PTB, birth prior to 37 completed weeks of gestation) or small-for-gestational age (SGA) infants born at term or a combination of both-preterm and SGA newborns. ${ }^{5}$ Various factors determine the length of gestation and intrauterine growth of the fetus and consequently the birth weight. Multiple causes of LBW have been identified, which include genetics, early labour, multiple pregnancy, various maternal illnesses (ie, pregnancy-induced hypertension, diabetes mellitus and infections), drug abuse (including tobacco and alcohol), maternal age, height, overweight and obesity along with deprived socioeconomic factors. ${ }^{6}$ Environmental factors, nutritional status and diet of the mother also affect fetal development. ${ }^{7}$ Alterations in the nutrient supply to the fetus result in nutrient deprivation, causing restricted growth. ${ }^{7}$

LBW is a major underlying cause of infant mortality and childhood morbidity. ${ }^{8}$ Additionally, there is a clear association between LBW and increased risk for many diseases later in life, such as metabolic syndrome, diabetes mellitus type 2, cardiovascular diseases, hypertension or cancer. ${ }^{9-11}$ Hence, early interventions starting before or during pregnancy have the potential to prevent LBW and decrease the risks for adverse health outcomes of LBW infants.

In 2012, the World Health Assembly agreed on six global nutrition targets including a 30\% reduction of the LBW rate by 2025. ${ }^{12}$ A conceptual framework for nutrition-specific and nutrition-sensitive interventions has been proposed. ${ }^{13}$ Nutrition-specific interventions are those that address the immediate determinants of nutrition such as supplementation with essential vitamins and minerals, while nutrition-sensitive interventions are those that address the underlying causes of undernutrition, for example, malaria prevention in pregnant women. ${ }^{14}$ In this overview of systematic reviews, we aimed to summarise the evidence of nutrition-specific and nutrition-sensitive interventions to prevent LBW.

\section{METHODS}

\section{Search strategy and selection criteria}

We searched the following electronic databases in September 2015 in order to identify systematic reviews that assessed the effects of interventions to prevent LBW: MEDLINE, EMBASE, CINAHL and the Cochrane
Database of Systematic Reviews using related thesaurus terms and a wide range of keywords. Additional journals and proceedings of major conferences were handsearched. The reference lists of included articles and current guidelines were checked for further articles not identified elsewhere. Details on the search strategies can be found in online supplementary tables 1-3. No limits were applied to the databases either in terms of publication date or language.

Systematic reviews of randomised controlled trials (RCTs) focusing on interventions to reduce the risk of LBW or SGA, either directly or indirectly, were eligible for inclusion. As antenatal interventions to reduce PTB were recently reviewed, we did not include reviews that focused only on PTB. ${ }^{15}$ Nutrition-specific and nutrition-sensitive interventions periconceptionally and/or during pregnancy were considered for inclusion. We included reviews where participants were women with a singleton pregnancy despite any potential risk of LBW. Reviews where the study population comprised women with singleton and multiple pregnancies were only included if data were presented separately. We excluded reviews that only selected women with a multiple pregnancy. We included reviews with RCTs and quasi-RCTs. Reviews that included other study designs were not eligible for inclusion as well as reviews not reporting on LBW or SGA. In the case we only identified Cochrane reviews, we did not search for further reviews on the particular topic because of the high quality of Cochrane reviews. In the absence of a Cochrane review, other systematic review formats were considered for inclusion.

\section{Data extraction and management}

Titles and abstracts identified by the search were independently screened for inclusion by two review authors using the systematic review software EPPI-Reviewer $4 .{ }^{16}$ Two authors independently assessed full texts for eligibility against the prespecified inclusion criteria. In order to identify all relevant systematic reviews that focus on nutritional interventions which aim to reduce LBW, we reviewed methods, study population and outcomes. Data from reviews were independently extracted by two authors using a predesigned data extraction form. We resolved discrepancies through discussion with a third author.

\section{Assessment of methodological quality}

Two authors independently assessed the methodological quality of the included reviews using A Measurement Tool to Assess Reviews (AMSTAR). ${ }^{17}$ Discrepancies were resolved through discussion. AMSTAR uses 11 distinct questions to evaluate the methods used in the systematic review (see online supplementary table 4). Each question is answered as yes (clearly done), no (clearly not done), cannot answer or not applicable. Each 'yes' response corresponded to one point and the total number of points was summed per row. Scores of 8-11 points were considered as high quality, $4-7$ as moderate 
quality and less than 3 as low methodological quality reviews (table 1 ).

\section{Outcomes}

The primary outcome was LBW, defined as weight at birth less than $2500 \mathrm{~g}$, regardless of the gestational age of the infant. Secondary outcomes included VLBW (less than $1500 \mathrm{~g}$ ), ELBW (less than $1000 \mathrm{~g}$ ), SGA (birth weight below the 10th percentile of gestational age), intrauterine growth restriction (IUGR) and PTB (less than 37 weeks of gestation).

\section{Data synthesis}

We compiled the information and results of the individual systematic reviews in a narrative way. 'Characteristics of included systematic reviews' table include the date of search, number of studies and participants, objective, study design, type of participants, interventions and comparison, outcomes and summary of quality of included studies (see online supplementary table 5) and 'Characteristics of interventions' table include low-risk or high-risk population, dose, frequency and start of intervention (table 2). We categorised similar interventions together creating nine groups: supplementation with vitamins, minerals, MMNs, proteins, marine oil and fatty acids, nutritional education, reduced salt intake, deworming and preventive antimalarial drugs. Results are presented according to intervention type. We limited the control group to placebo, no intervention or absence of the intervention. Summary estimates from individual studies presented as relative risk and OR with 95\% CI are reported (see online supplementary tables 6-14). This overview review was registered at the International Prospective Register of Systematic Reviews (PROSPERO), number CRD42015024814 (24 July 2015).

\section{RESULTS}

\section{Search results}

Our search identified 909 records. Another 159 references were added after handsearch. We excluded 131 duplicates and screened title and abstract of 937 publications. A total of 260 full-text articles were assessed for inclusion. After exclusion of 231 articles, 29 reviews were included. Another six reviews were excluded after further assessment of the full-texts. ${ }^{18-23}$ Characteristics and reason for exclusion of these studies are described in online supplementary table 15 . Finally, 23 systematic reviews met our inclusion criteria and were included into the qualitative synthesis of this overview review (figure 1).

\section{Characteristics of included studies}

All included systematic reviews assessed the effect of nutrition-specific and nutrition-sensitive interventions in pregnancy on maternal, fetal, neonatal and infant outcomes (see online supplementary table $5)$. Twenty-one reviews reported on $\mathrm{LBW},{ }^{24-44} 11$ on SGA $^{29}$ 31-33 35-39 4546 and 3 reviews on IUGR. ${ }^{28} 4546$ Two studies reported $\mathrm{VLBW}^{30}{ }^{32}$ while none of the included reviews reported ELBW. Twenty-two studies assessed the effect of the interventions on PTB. ${ }^{24-43} 4546$ All systematic reviews included individually RCTs $^{24-46} 9$ cluster-RCTs ${ }^{24} 30-323536384245$ and 10 both RCTs and quasiRCTs. ${ }^{24} 26$ 29-32 43-46 The number of included trials per review ranged from $2^{41}$ to 61 trials. ${ }^{30}$ The number of included participants in the reviews ranged from 603 participants ${ }^{41}$ to over 310000 participants. ${ }^{24}$ Supplementation with vitamins was reported in five reviews, $24-264546$ supplementation with minerals in eight reviews ${ }^{27-34}$ and MMN supplementation was assessed in three reviews. ${ }^{35-37}$ There was one review on protein supplementation and nutritional education ${ }^{38}$ and two reviews reported on marine oil and fatty acid supplementation. ${ }^{39} 40$ One review evaluated reduced salt intake, ${ }^{41}$ one deworming ${ }^{42}$ and two reviews preventive antimalarial drugs. ${ }^{434}$

\section{Methodological quality of the included reviews}

Sixteen reviewswere of high quality. ${ }^{24-2628-3336383942434546} \mathrm{Six}$ reviews were assessed to be of moderate quality. ${ }^{273437404144}$ Only one included review had a score of three and, therefore, was judged to be of low methodological quality (table 1)..$^{35}$

\section{Effects of interventions}

Oral supplementation with vitamins alone or in combination

Five systematic reviews investigated the effect of oral supplementation with vitamin $\mathrm{A},{ }^{24}$ vitamin $\mathrm{C}^{45}$ or vitamin E during pregnancy ${ }^{46}$ and oral folic acid supplementation $^{2526}$ during the periconceptional period and pregnancy (see online supplementary table 6 ).

\section{Vitamin A}

A review including 19 RCTs, quasi-RCTs and cluster-RCTs randomising over 310000 pregnant women living in areas with endemic vitamin A deficiency or in areas with adequate intake assessed the effect of vitamin A supplementation or one of its derivatives alone or in combination with other supplements on LBW and PTB. ${ }^{24}$ Vitamin A alone compared with placebo or no treatment did not reduce LBW or PTB. Vitamin A with other micronutrients (iron + folate) versus micronutrient supplements without vitamin A significantly reduced LBW by $33 \%$, but did not affect PTB.

\section{Vitamin C}

Vitamin C supplementation alone or in combination with other micronutrients compared with placebo, no placebo or other micronutrients was assessed in one review that included 29 RCTs and quasi-RCTs randomising 24300 pregnant women. ${ }^{45}$ No significant differences in PTB, SGA or IUGR could be found between groups. LBW was not reported.

\section{Vitamin $E$}

Twenty-one RCTs and quasi-RCTs randomising 22129 pregnant women living in areas with inadequate vitamin $\mathrm{E}$ intake or areas with presumed adequate intake were included in this review. ${ }^{46}$ Any vitamin E supplementation 


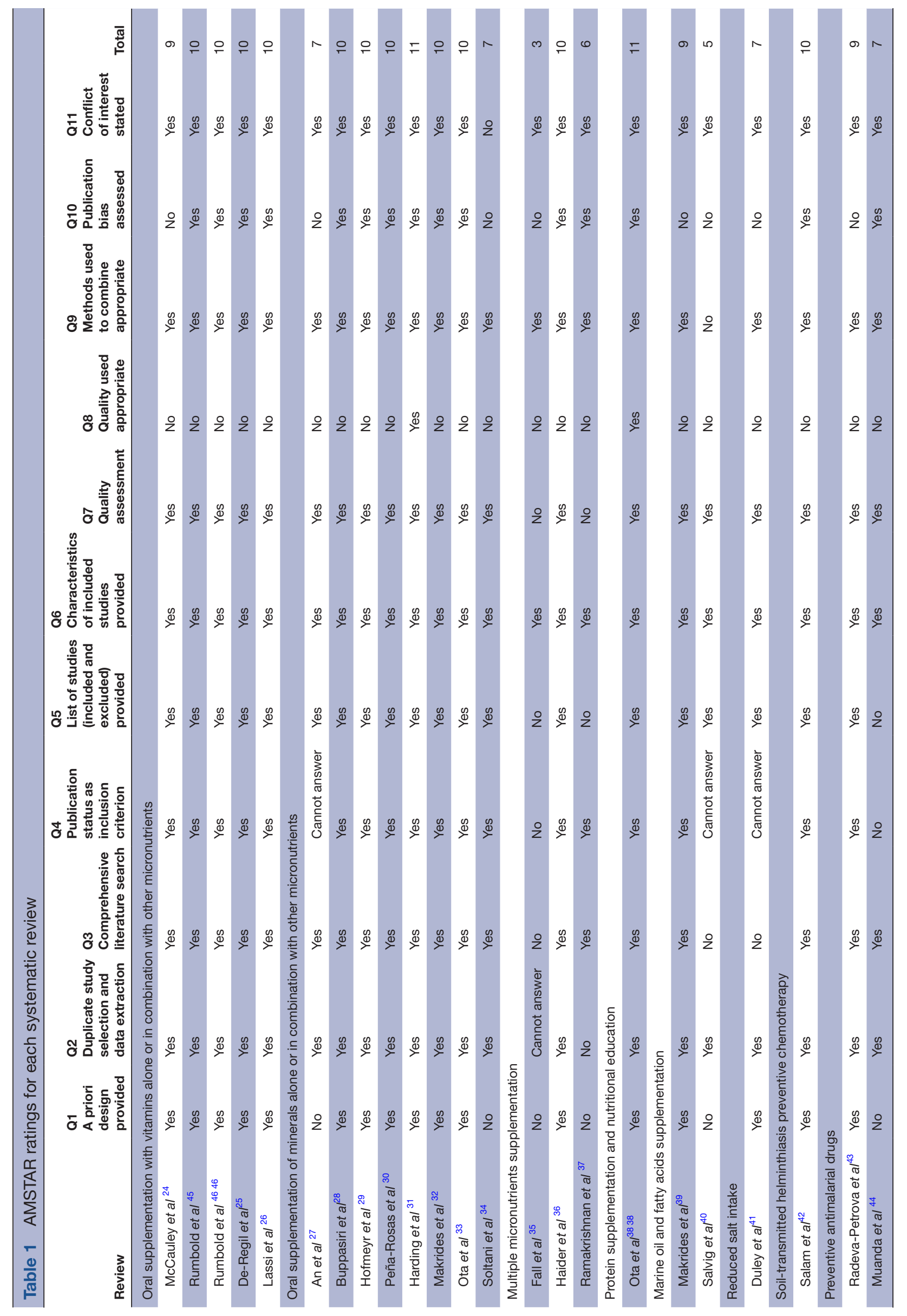

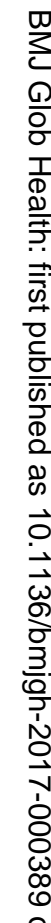

○े वे

응

造 흥 \&

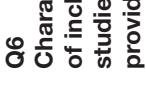

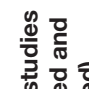

范

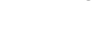

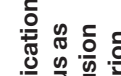

吅昰焉. 

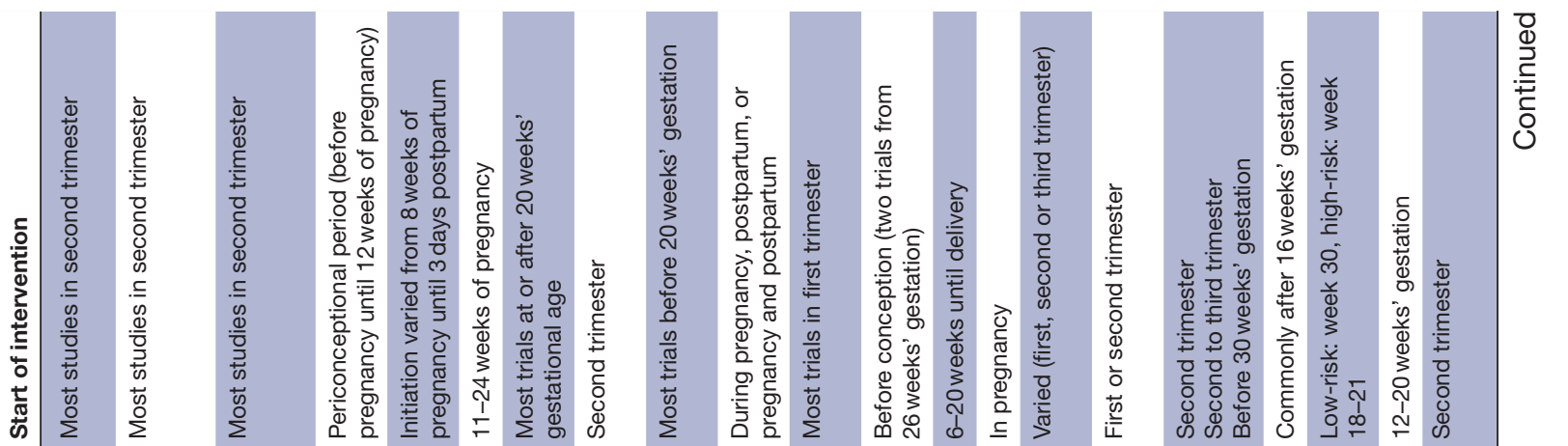

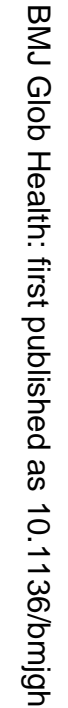

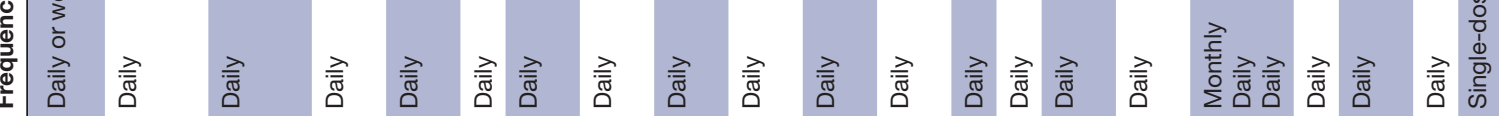

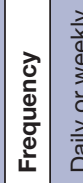

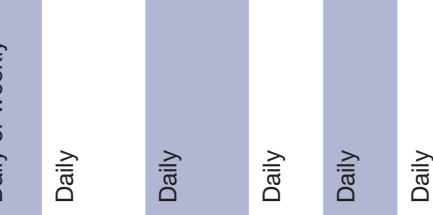
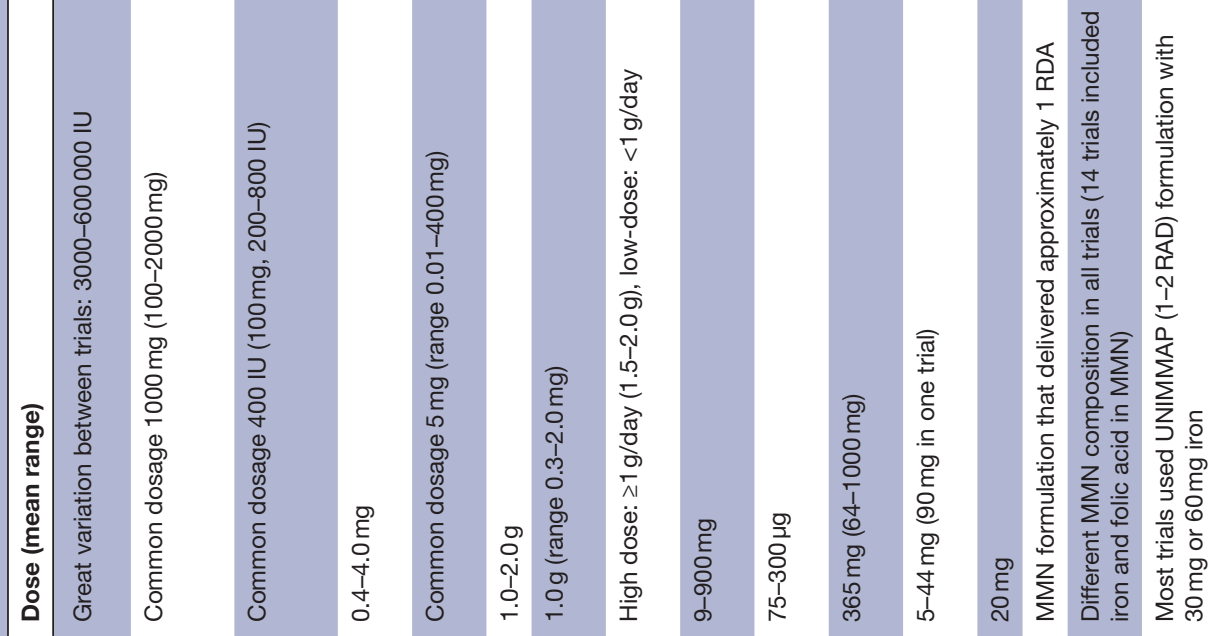

站

$\frac{\pi}{\frac{1}{9}} \frac{\pi}{\frac{\pi}{2}}$

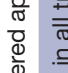

离竞

茫垔

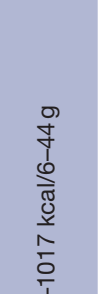

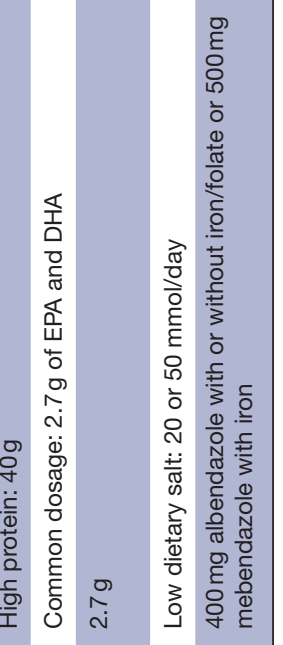

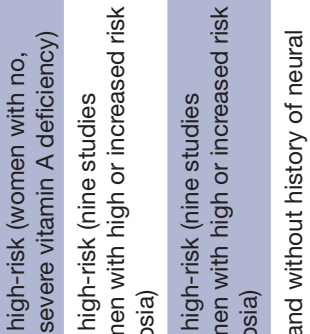

ᄃ)

들

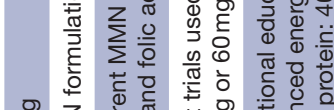

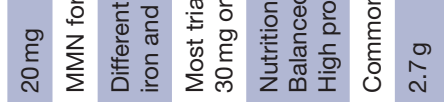

종

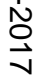

ठे

운

N

(D)

$\frac{0^{\circ}}{3}$

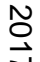

뭉

$\sum^{\circ}$

$\frac{1}{\infty}$<smiles>CCCCCCCC</smiles>

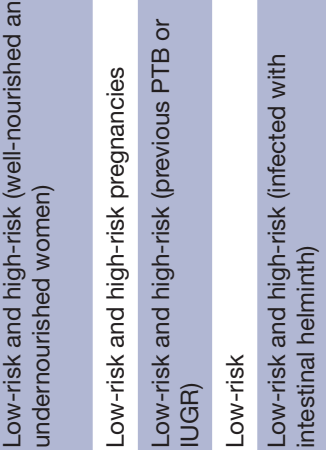




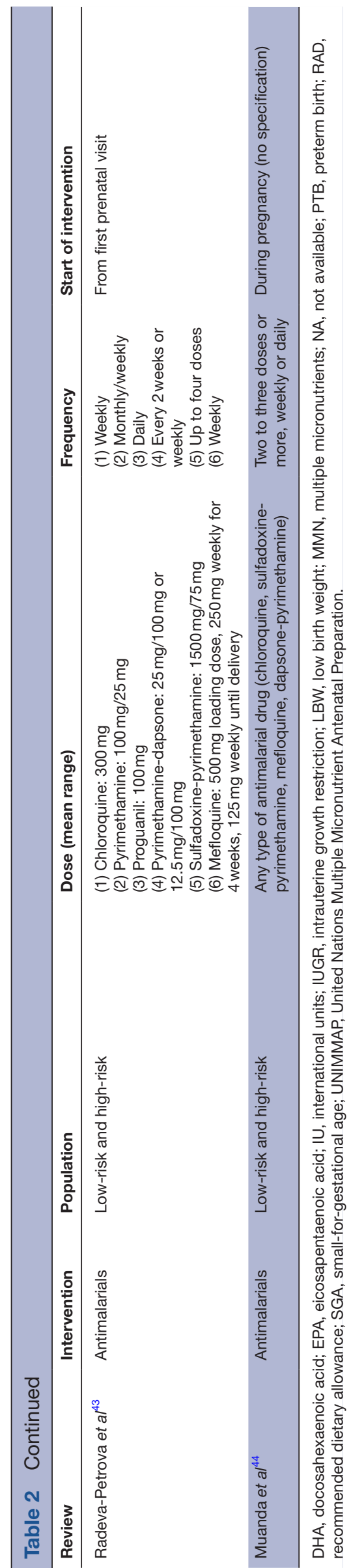

did not reduce PTB, SGA or IUGR. LBW was not reported as an outcome.

\section{Folic acid}

Five RCTs randomising 7391 women who become pregnant or were less than 12 weeks pregnant were supplemented with folic acid. ${ }^{25}$ Folic acid supplementation was compared with no intervention, placebo or other micronutrients without folic acid. Another review included 31 RCTs and quasi-RCTs randomising 17771 pregnant women and evaluated the effect of folic acid supplementation alone or with other micronutrients versus no folic acid. ${ }^{26}$ In both studies, folic acid supplementation did not alter the risk of LBW or PTB.

Oral supplementation of minerals alone or in combination with other micronutrients

Eight reviews assessed the effect of mineral supplementation during pregnancy on maternal and infant outcomes. Interventions included calcium, ${ }^{27-29}$ iron, ${ }^{30}$ iodine,${ }^{31}$ magnesium ${ }^{32}$ and zinc ${ }^{33}{ }^{34}$ supplementation (see online supplementary table 7).

\section{Calcium}

One review included four RCTs involving 14524 pregnant women without diseases such as hypertension, diabetes mellitus or renal diseases who received oral calcium supplementation during pregnancy. ${ }^{27}$ No positive effects on LBW and PTB were observed. Another review compared pregnancy outcomes of 18587 randomised women from 25 RCTs receiving oral calcium supplementation (other than for preventing or treating hypertension) with women receiving placebo or no treatment. ${ }^{28}$ No statistically significant group differences were observed for LBW, PTB or IUGR. An additional review investigated the effect of calcium supplementation during pregnancy for preventing hypertension disorders and related problems in 24 RCTs and quasi-RCTs randomising 17964 pregnant women. ${ }^{29}$ Routine oral high-dose calcium ( $\geq 1 \mathrm{~g} /$ day $)$ supplementation did not alter the risk of LBW and SGA, but decreased PTB by $24 \%$. However, oral low-dose calcium $(<1 \mathrm{~g} /$ day $)$ supplementation significantly reduced LBW by $80 \%$, but had no effect on the risk of PTB and SGA.

Iron

One review of 61 RCTs, quasi-RCTs and cluster-RCTs randomising 43274 pregnant women assessed the effect of daily iron supplementation on LBW, VLBW and PTB. ${ }^{30}$ Oral iron supplementation, iron plus folic acid or iron plus other vitamins and minerals had no effect on the investigated outcomes.

\section{lodine}

One review including 14 RCTs, quasi-RCTs and cluster RCTs randomising over 2700 women assessed the benefits and harms of iodine supplementation during preconception, pregnancy or the postpartum period. ${ }^{31}$ There was no evidence of an effect of any supplement containing 


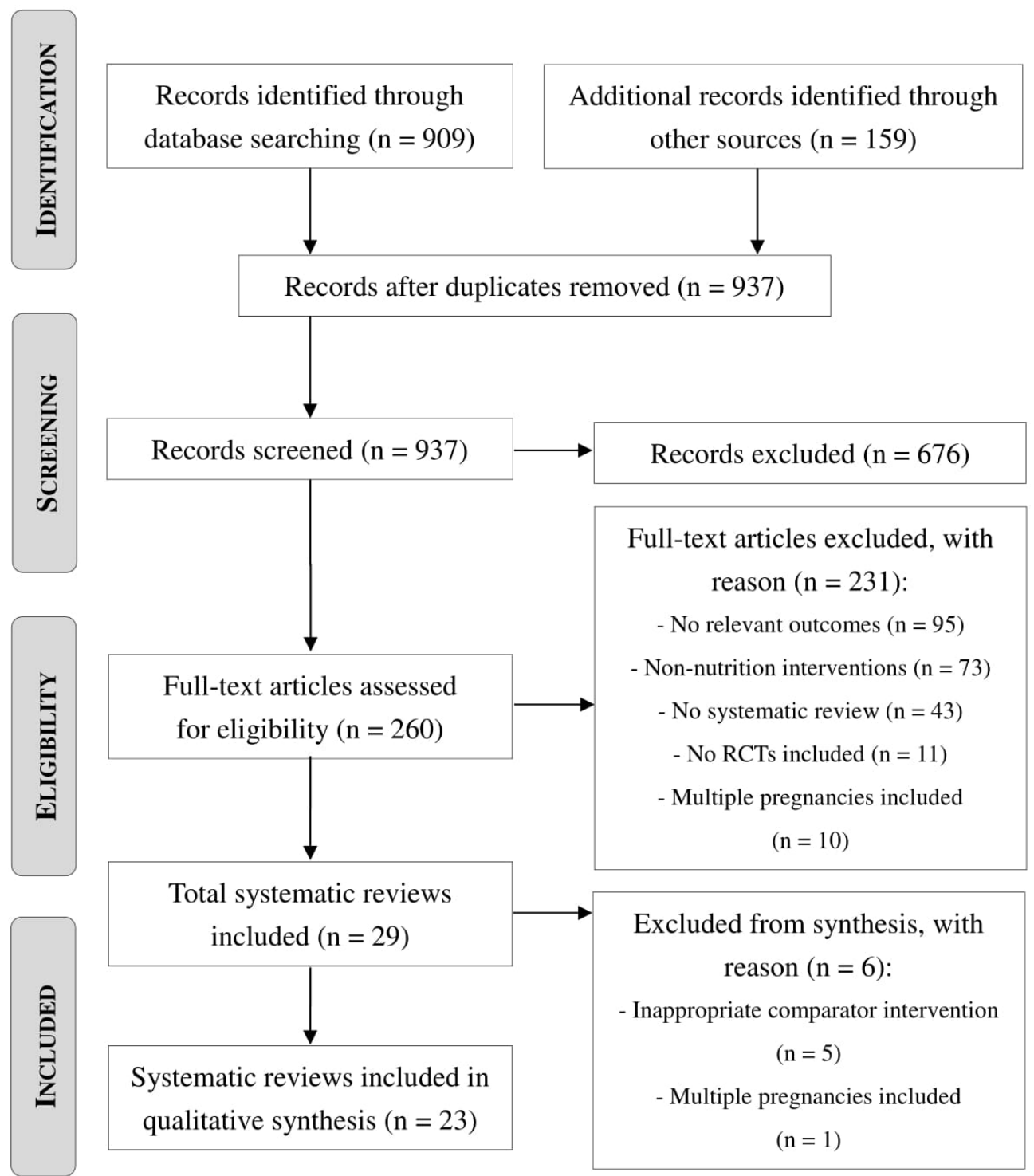

Figure 1 Flow diagram for literature search (PRISMA). RCTs, randomised controlled trials.

iodine versus the same supplement without iodine or no intervention/placebo for the reported outcomes LBW, PTB and SGA.

\section{Magnesium}

A review including 10 RCTs, quasi-RCTs and cluster-RCTs randomising 9090 pregnant women with normal or highrisk pregnancies assessed the effect of oral magnesium supplementation. ${ }^{32}$ There was no evidence of a difference for LBW, VLBW, PTB and SGA between women receiving magnesium compared with no magnesium supplementation.

Zinc

Zinc supplementation during pregnancy was assessed in a review randomising over 17000 pregnant women from 21 RCTs. ${ }^{33}$ The intervention resulted in a $14 \%$ reduction of PTB while LBW and SGA were unaffected. Another review investigated the effect of zinc supplementation in adolescent pregnancy from five RCTs involving 1855 adolescent pregnant women. ${ }^{34}$ Oral zinc supplementation reduced the likelihood of LBW by $61 \%$, but no significant difference in PTB was observed.

\section{Multiple micronutrient supplementation}

The effect of MMN supplementation on LBW, PTB and SGA was assessed in three reviews (see online supplementary table 8). ${ }^{35-37}$ MMN supplementation provided one recommended daily nutrient intake of vitamin $A$, vitamin $B_{1}$, vitamin $B_{2}$, niacin, vitamin $B_{6}$, vitamin $\mathrm{B}_{12}$, folic acid, vitamin $\mathrm{C}$, vitamin $\mathrm{D}$, vitamin $\mathrm{E}$, copper, selenium and iodine with iron and zinc to pregnant women. ${ }^{47} \mathrm{~A}$ significant reduction of LBW in the range of $11 \%-14 \%$ and a significant reduction of SGA in the range of $10 \%-17 \%$ could be observed following MMN supplementation in all three reviews. However, when comparing MMN containing iron and folic acid versus placebo, the effect was not statistically significant. ${ }^{36}$ The risk of PTB was unaffected in all studies. 


\section{Protein supplementation}

One review including 17 RCTs and cluster-RCTs randomising 9030 pregnant women assessed the effects of balanced energy and protein intake on pregnancy outcomes (see online supplementary table 9). ${ }^{38}$ While balanced protein/energy supplementation compared with no intervention in pregnancy resulted in a reduction of SGA by $21 \%$, high protein supplementation compared with low or no protein supplementation increased the likelihood of SGA by $58 \%$. No effects on PTB were seen in both interventions and LBW was not assessed.

\section{Marine oil and fatty acid supplementation}

One review with six RCTs randomising 2755 pregnant women investigated the effect of oral marine oil (fish or algal oils) and other prostaglandin precursor supplementation during pregnancy and found no evidence of a significant difference for LBW, PTB and SGA (see online supplementary table 10). ${ }^{39}$ Another review including three RCTs randomising 1187 singleton pregnant women compared LBW and PTB between women receiving longchain n-3 fatty acid supplementation with placebo and found a $39 \%$ reduction of PTB but no effect on the risk of LBW. ${ }^{40}$

\section{Nutritional education}

One review randomising 9030 women from 17 RCTs and cluster-RCTs focused on antenatal dietary education to improve maternal and infant health outcomes. ${ }^{38}$ LBW was reduced by $96 \%$ and PTB by $54 \%$ for women receiving nutritional education to increase energy and protein intake compared with no nutritional education in pregnancy. No effect on SGA was seen for this intervention (see online supplementary table 11).

\section{Reduced salt intake}

In one review with two RCTs and 603 women, the effects of low salt intake (20 or $50 \mathrm{mmol} /$ day) compared with an unchanged diet on the risk of pre-eclampsia and its consequences was evaluated. ${ }^{41}$ There was no evidence of an effect on LBW or PTB (see online supplementary table 12).

\section{Soil-transmitted helminthiasis preventive chemotherapy}

One review involving four RCTs and cluster-RCTs randomising 4265 pregnant women in the second and third trimester assessed the effect of antihelminthics administration during pregnancy on maternal anaemia and pregnancy outcomes. ${ }^{42}$ No effects on the risk of LBW or PTB were seen for antihelminthics administration compared with control (see online supplementary table 13).

\section{Preventive antimalarial drugs}

Two systematic reviews with 25 (37981 women) ${ }^{44}$ and 17 (14481 women) ${ }^{43}$ RCTs and quasi-RCTs evaluated the effect of antimalarial drugs for preventing malaria during pregnancy and the risk of LBW (see online supplementary table 14). Both reviews found a $27 \%$ reduction of
LBW for women receiving antimalarial drugs compared with women not receiving these drugs during pregnancy. The intervention had no effect on the risk of PTB and other outcomes were not reported. ${ }^{43}$

\section{DISCUSSION}

This overview evaluated the effects of nutrition-specific and nutrition-sensitive interventions on LBW. Overall, among 34 comparisons from 23 systematic reviews, six interventions reduced the incidence of LBW and none of the interventions significantly increased the rates of LBW. Two interventions reduced the risk of SGA, while one significantly increased the incidence of SGA. PTB was shown to be reduced in response to four interventions. VLBW and IUGR were unaffected by these interventions.

Vitamin A supplementation with other micronutrients (iron + folate) compared with micronutrient supplementation without vitamin A had a positive effect and reduced the risk of LBW by $33 \% .{ }^{24}$ However, these findings are from a study involving only HIV-positive women and when comparing vitamin A supplementation alone versus placebo or no treatment, a reduction of LBW could not be observed. Other single vitamin supplementations during pregnancy did not show any benefits for pregnancy outcomes such as LBW, PTB, SGA or IUGR.

In relation to mineral supplementation during pregnancy, calcium and zinc were effective to improve maternal and infant outcomes. Calcium supplementation during pregnancy for preventing hypertensive disorders and related problems led to $80 \%$ reduction of LBW for women receiving low-dose calcium (less than $1 \mathrm{~g}$ per day) ${ }^{29}$ However, this observation was obtained from two trials with only 134 women. On the other hand, highdose calcium (at least $1 \mathrm{~g} /$ day) supplementation during pregnancy reduced the risk of PTB by $24 \%$ (high GRADE quality) but had no effect on LBW. ${ }^{29}$ The findings on calcium supplementation are inconsistent with two other reviews included in this overview which also investigated the effect of calcium supplementation on pregnancy outcomes. ${ }^{27} 28$ In both studies, the majority of included trials used high-dose calcium supplementation but did not observe positive effects on PTB or LBW. Reviews differed in eligibility criteria for trials and studies varied in the number of included risks groups (eg, high risk for pre-eclampsia), timing of calcium supplementation or dietary calcium intake, which might explain differences in observations. ${ }^{27-29}$

Zinc supplementation resulted in a $61 \%$ reduction in LBW in one review focusing on pregnancy outcomes in adolescent pregnancy. ${ }^{34}$ The result is in contrast to the Cochrane review evaluating zinc supplementation during pregnancy. ${ }^{33}$ This review did not find a reduction of LBW rates but demonstrated that the intervention reduced the risk of PTB by $14 \%$ (moderate GRADE quality). Most of the included studies were conducted in low-income countries, assuming overall poor nutrition 
status. The authors concluded that supplementation with a combination of vitamins and minerals will improve maternal and infant health rather than particular nutrients alone. ${ }^{33}$ This assumption can be supported by findings from studies on MMN supplementation during pregnancy. All included reviews on MMN supplementation demonstrated a positive effect on the risk of LBW (reduction ranged from $10 \%$ to $14 \%$ ) and SGA (reduction ranged from $10 \%$ to $17 \%) \cdot{ }^{35-37}$ In Haider et al, the quality of evidence for reduction of LBW was high and for reduction of SGA moderate. ${ }^{36}$ The authors suggested that MMN supplementation improved women's overall nutritional status and consequently reduces women's risk for maternal infections due to a stable state of health and immune system. ${ }^{36}$

Another review concluded that protein and energy supplementations contribute to an overall improvement of women's nutritional status and thereby decrease the risk of adverse pregnancy outcomes. ${ }^{38}$ In this review, balanced protein/energy supplementation significantly reduced the risk of SGA by $21 \%$ (moderate GRADE quality) ${ }^{38}$ On the other hand, high protein supplementation compared with low or no protein supplementation was associated with a $58 \%$ increased risk of SGA (moderate GRADE quality) ${ }^{38}$ Although these findings are from analysis involving one study with a small population, it gives an indication that high protein supplementation alone might be potentially harmful for pregnant women. A link between high protein intake during pregnancy and adverse birth outcomes has also been shown by other epidemiological studies. ${ }^{48}{ }^{49}$ Animal studies suggest that high-protein exposure during development reprograms the fetus' bodyweight and results in defects in energy expenditure. ${ }^{50}$ However, the mechanism is still not well understood.

A review investigating the effect of marine $n-3$ fatty acids on the prevention of PTB and preterm labour found a $39 \%$ reduction of PTB but no effect on LBW. ${ }^{40}$ Although there was a beneficial effect, the review concluded that general recommendations could not be given based on their finding due to the limited number of included studies and conflicting results from other studies. ${ }^{40}$

Nutritional education appeared highly effective in reducing the risk of LBW (96\%) and PTB (54\%, low GRADE quality); however, the evidence was derived from only one (Bangladesh) and two (rural area in Greece and low-income African-American women in USA) studies, respectively. ${ }^{38}$ Nutritional education to increase women's protein intake consisted of education sessions, nutrition counselling or in-home nutrition assessment and counselling visits. Findings suggest that this intervention may be especially beneficial for undernourished pregnant women, but results have to be interpreted with caution due to the limited quality of included trials. ${ }^{38}$ This intervention seems promising, but further high-quality studies are needed to confirm the impact of nutritional education on LBW and PTB risk reduction.
Pregnancy increases the risk of malaria infection and in return, malaria infection is associated with an increased risk of LBW. ${ }^{51}$ Consistently, two included reviews could show that successful prevention of malaria infection using antimalarial drugs during pregnancy significantly reduced the incidence of LBW by $27 \% .{ }^{43}$ In Radeva-Petrova $e t a l$, the quality of evidence for reducing the risk of LBW was moderate. ${ }^{43}$

A previous overview review focused on antenatal interventions to prevent PTB. ${ }^{15}$ Our results are consistent with this review with two exceptions. Piso et al found that vitamin $\mathrm{C}$ supplementation during pregnancy increased the risk of PTB and magnesium supplementation decreased the risk of PTB, LBW and SGA. ${ }^{15}$ However, the Cochrane reviews from which data were extracted had previously been updated and new studies had been added resulting in changed conclusions. Currently, there is no evidence that magnesium or vitamin $\mathrm{C}$ supplementation during pregnancy positively or negatively affect the risk of PTB, LBW or SGA.

Included trials in individual reviews varied widely and were from low-income, middle-income and high-income countries. Except one,$^{35}$ no other review focused exclusively on developing or developed countries. Furthermore, types of participants were not restricted to maternal nutrition status and no review categorised women according to their body mass index (BMI); hence limiting generalisability of the findings. Only three reviews performed subgroup analysis to evaluate the effect of interventions for women with different nutrition status. ${ }^{33} 3638$ For example, MMN supplementation significantly reduced the risk of PTB for women with lower BMI but not among those with higher BMI. ${ }^{36}$ It seems reasonable to suggest that the effect of nutritional interventional depends on women's nutrition status. Therefore, further research should address nutritional interventions in various populations (ie, undernourished vs adequately nourished vs overweight/obese women).

The majority of included reviews were of high methodological quality. Although some interventions showing a positive effect on reducing LBW were reported in reviews that included more than 12 trials, in most cases findings on LBW were derived from one or two studies. In addition, different reviews investigating the effect of the same intervention on pregnancy outcomes were unable to show the same the effect. This may be explained by differences in eligibility criteria of potential studies and study populations, except MMN supplementation and preventive antimalarial drugs during pregnancy which led to a significant reduction of LBW in all included systematic reviews. Based on available evidence, WHO has formulated recommendations for intermittent preventive treatment of pregnant women for malaria. ${ }^{52}$ MMN supplementation might be considered for policy development regarding routine administration during pregnancy to improve maternal and infant health. Other interventions need further assessment before routine implementation can be recommended. 
We intended to summarise the evidence from multiple systematic reviews and to our knowledge, this is the first overview review focusing on nutrition interventions to prevent or reduce the risk of LBW. Our results and conclusions are mainly affected by the quality of systematic reviews and their included studies. The completeness of interventions included in our overview is determined by the availability of reviews addressing nutrition-specific and nutrition-sensitive interventions to improve pregnancy outcomes. Furthermore, we limited the search to the main databases and acknowledge that there is a possibility that we missed systematic reviews included only in other literature databases.

In conclusion, here we presented a comprehensive compilation of mostly nutrition-specific and some nutrition-sensitive interventions aimed to reduce LBW. This opens the opportunity to primarily consider nutrition interventions as part of the efforts to reach the global target to decrease the rate of LBW by $30 \%$, as committed to by the WHO Member States in 2012. Affordable, accessible and appropriate antenatal care with effective nutrition interventions is critical for preventing LBW. ${ }^{12}$

Acknowledgements We thank and Yuko Serizawa and Chiemi Kataoka for designing the database search strategies, conducting the systematic search and retrieving the full-texts.

Contributors KdSL and EO were responsible for the study conception and design. KdSL (all), AD (half) and OOB (half) screened studies for eligibility. KdSL and PS extracted data from reviews and assessed methodological quality. KdSL wrote the paper. All authors made critical revisions to the manuscript, read and approved the final version.

Funding Nutrition International and the Evidence and Programme Guidance, Department of Nutrition for Health and Development, WHO provided partial internal or external funding for this work. WHO acknowledges the financial support of Bill \& Melinda Gates Foundation to build the evidence base on systematic reviews on the effects of nutrition actions for health and development. The source of funding had no role in study design, data collection and interpretation and writing of the overview review. The corresponding author EO had full access to all data and had final responsibility for the decision to submit this review for publication.

Disclaimer The authors alone are responsible for the views expressed in this article and they do not necessarily represent the views, decisions or policies of the institutions with which they are affiliated.

Competing interests JPP-R, EO, LMD-R and RM are authors and/or co-authors of included Cochrane reviews. They were not involved in the screening process or selection of reviews for inclusion. All other authors declare that they have no conflicts of interests.

Provenance and peer review Not commissioned; externally peer reviewed.

Open Access This is an Open Access article distributed in accordance with the Creative Commons Attribution Non Commercial (CC BY-NC 4.0) license, which permits others to distribute, remix, adapt, build upon this work non-commercially, and license their derivative works on different terms, provided the original work is properly cited and the use is non-commercial. See: http://creativecommons.org/ licenses/by-nc/4.0/

C Article author(s) (or their employer(s) unless otherwise stated in the text of the article) 2017. All rights reserved. No commercial use is permitted unless otherwise expressly granted.

\section{REFERENCES}

1. United Nations Children's Fund. Undernourishment in the womb can lead to diminished potential and predispose infants to early death 2014 http://www.data.unicef.org/nutrition/low-birthweight.html (accessed 29 Jun 2017).
2. WHO. International statistical classification of diseases and related health problems (ICD-10. 10 ed. Geneva: World Health Organization, 2010.

3. Kramer MS. Determinants of low birth weight: methodological assessment and meta-analysis. Bull World Health Organ 1987;65:663-737.

4. Eichenwald EC, Stark AR. Management and outcomes of very low birth weight. N Engl J Med 2008;358:1700-11.

5. Villar J, Papageorghiou AT, Knight HE, et al. The preterm birth syndrome: a prototype phenotypic classification. Am J Obstet Gynecol 2012;206:119-23.

6. Mayer C, Joseph KS. Fetal growth: a review of terms, concepts and issues relevant to obstetrics. Ultrasound Obstet Gynecol 2013;41:136-45.

7. Cetin I, Mandò $C$, Calabrese $S$. Maternal predictors of intrauterine growth restriction. Curr Opin Clin Nutr Metab Care 2013;16:310-9.

8. Lawn JE, Cousens S, Zupan J. Lancet Neonatal Survival Steering Team. 4 million neonatal deaths: when? where? why? Lancet 2005;365:891-900

9. Reyes L, Manalich R. Long-term consequences of low birth weight. Kidney Int 2005;68:S107-S111.

10. Barker DJ. The developmental origins of adult disease. J Am Coll Nutr 2004;23(6 Suppl):588S-95.

11. Fall $\mathrm{CH}$. Fetal programming and the risk of noncommunicable disease. Indian J Pediatr 2013;80 (suppl 1):13-20.

12. WHO. Global nutrition targets 2025: low birth weight policy brief WHO/NMH/NHD/14.5. Geneva : World health organization, 2014.

13. Maternal and child nutrition. Executive summary of the lancet maternal and child nutrition series. The Lancet 2013.

14. Ruel MT, Alderman H; Maternal and Child Nutrition Study Group. Nutrition-sensitive interventions and programmes: how can they help to accelerate progress in improving maternal and child nutrition? Lancet 2013;382:536-51.

15. Piso B, Zechmeister-Koss I, Winkler R. Antenatal interventions to reduce preterm birth: an overview of Cochrane Systematic Reviews. BMC Res Notes 2014;7:265.

16. Thomas J, Brunton J, Graziosi S. EPPI-Reviewer 4.0: software for research synthesis. EPPI-Centre Software London: Social Science Research Unit: Institute of education, university of London, 2010.

17. Shea BJ, Grimshaw JM, Wells GA, et al. Development of AMSTAR: a measurement tool to assess the methodological quality of systematic reviews. BMC Med Res Methodol 2007;7:10.

18. De-Regil LM, Palacios C, Lombardo LK, et al. Vitamin D supplementation for women during pregnancy. Cochrane Database Syst Rev 2016(1):CD008873.

19. Muktabhant B, Lawrie TA, Lumbiganon P, et al. Diet or exercise or both, for preventing excessive weight gain in pregnancy. Cochrane Database Syst Rev 2015;6.

20. Peña-Rosas JP, De-Regil LM, Gomez Malave H, et al. Intermittent oral iron supplementation during pregnancy. Cochrane Database Syst Rev 2015;10:CD009997.

21. Say L, Gülmezoglu AM, Hofmeyr GJ. Maternal nutrient supplementation for suspected impaired fetal growth. Cochrane Database Syst Rev 2003;1:CD000148.

22. Thangaratinam S, Rogozińska E, Jolly K, et al. Effects of Interventions in Pregnancy on Maternal Weight and Obstetric Outcomes. Obstet Gynecol Surv 2012;67:e2088:603-4.

23. Kayentao K, Garner P, van Eijk AM, et al. Intermittent preventive therapy for malaria during pregnancy using 2 vs 3 or more doses of sulfadoxine-pyrimethamine and risk of low birth weight in Africa: systematic review and meta-analysis. JAMA 2013;309:594-604.

24. McCauley ME, van den Broek N, Dou L, et al. Vitamin A supplementation during pregnancy for maternal and newborn outcomes. Cochrane Database Syst Rev 2015;10:CD008666.

25. De-Regil LM, Peña-Rosas JP, Fernández-Gaxiola AC, et al. Effects and safety of periconceptional oral folate supplementation for preventing birth defects. Cochrane Database Syst Rev 2015;12:CD007950.

26. Lassi ZS, Salam RA, Haider BA, et al. Folic acid supplementation during pregnancy for maternal health and pregnancy outcomes. The Cochrane Database of syst Rev 2013;3 :

27. An LB, Li WT, Xie TN, et al. Calcium supplementation reducing the risk of hypertensive disorders of pregnancy and related problems: A meta-analysis of multicentre randomized controlled trials. Int $J$ Nurs Pract 2015;21 (suppl 2):19-31.

28. Buppasiri P, Lumbiganon P, Thinkhamrop J, et al. Calcium supplementation (other than for preventing or treating hypertension) for improving pregnancy and infant outcomes. Cochrane Database Syst Rev 2015;2:CD007079.

29. Hofmeyr GJ, Lawrie TA, Atallah AN, et al. Calcium supplementation during pregnancy for preventing hypertensive disorders and related problems. Cochrane Database Syst Rev 2014;6:CD001059. 
30. Peña-Rosas JP, De-Regil LM, Garcia-Casal MN, et al. Daily oral iron supplementation during pregnancy. Cochrane Database of syst Rev 2015;7.

31. Harding KB, Peña-Rosas JP, Webster AC, et al. lodine supplementation for women during the preconception, pregnancy and postpartum period. Cochrane Database Syst Rev 2017;3((3)):CD011761.

32. Makrides M, Crosby DD, Bain E, et al. Magnesium supplementation in pregnancy. The Cochrane database of systematic reviews 2014;4.

33. Ota E, Mori R, Middleton P, et al. Zinc supplementation for improving pregnancy and infant outcome. Cochrane Database Syst Rev 2015;2:CD000230.

34. Soltani H, Duxbury Alexandra MS, Rundle R, et al. A systematic review of the effects of dietary interventions on neonatal outcomes in adolescent pregnancy. Evidence Based Midwifery 2015;13:29-34.

35. Fall $\mathrm{CH}$, Fisher DJ, Osmond $\mathrm{C}$, et al. Multiple micronutrient supplementation during pregnancy in low-income countries: a metaanalysis of effects on birth size and length of gestation. Food Nutr Bull 2009;30(4 Suppl):S533-S546.

36. Haider BA, Bhutta ZA. Multiple-micronutrient supplementation for women during pregnancy. The Cochrane database of systematic reviews 2015;11.

37. Ramakrishnan U, Grant FK, Imdad A, et al. Effect of multiple micronutrient versus iron-folate supplementation during pregnancy on intrauterine growth. Nestle Nutr Inst Workshop Ser 2013;74:53-62.

38. Ota E, Hori H, Mori R, et al. Antenatal dietary education and supplementation to increase energy and protein intake. Cochrane Database Syst Rev 2015;6:CD000032.

39. Makrides M, Duley L, Olsen SF. Marine oil, and other prostaglandin precursor, supplementation for pregnancy uncomplicated by preeclampsia or intrauterine growth restriction. Cochrane Database Syst Rev 2006;3:CD003402.

40. Salvig JD, Lamont RF. Evidence regarding an effect of marine $n-3$ fatty acids on preterm birth: a systematic review and meta-analysis. Acta Obstet Gynecol Scand 2011;90:825-38.
41. Duley L, Henderson-Smart DJ. Reduced salt intake compared to normal dietary salt, or high intake, in pregnancy. Cochrane Database of Syst Rev 1999;3.

42. Salam RA, Haider BA, Humayun Q, et al. Effect of administration of antihelminthics for soil-transmitted helminths during pregnancy. Cochrane Database Syst Rev 2015;6:CD005547.

43. Radeva-Petrova D, Kayentao K, ter Kuile FO, et al. Drugs for preventing malaria in pregnant women in endemic areas: any drug regimen versus placebo or no treatment. Cochrane Database Syst Rev 2014;10:CD000169.

44. Muanda FT, Chaabane S, Boukhris T, et al. Antimalarial drugs for preventing malaria during pregnancy and the risk of low birth weight: a systematic review and meta-analysis of randomized and quasirandomized trials. BMC Med 2015;13:193.

45. Rumbold A, Ota E, Nagata C, et al. Vitamin C supplementation in pregnancy. The Cochrane Database Syst Rev 2015;9.

46. Rumbold A, Ota E, Hori $\mathrm{H}$, et al. Vitamin $\mathrm{E}$ supplementation in pregnancy. Cochrane Database of syst Rev 2015;9:CD004069.

47. UNICEF. Composition of a multi-micronutrient supplement to be used in pilot programmes among pregnant women in developing countries, 1999.

48. Sloan NL, Lederman SA, Leighton J, et al. The effect of prenatal dietary protein intake on birth weight. Nutr Res 2001;21(12):129-39.

49. Andreasyan K, Ponsonby AL, Dwyer T, et al. Higher maternal dietary protein intake in late pregnancy is associated with a lower infant ponderal index at birth. Eur J Clin Nutr 2007;61:498-508.

50. Daenzer M, Ortmann S, Klaus S, et al. Prenatal high protein exposure decreases energy expenditure and increases adiposity in young rats. J Nutr 2002;132:142-4.

51. Desai M, ter Kuile FO, Nosten F, et al. Epidemiology and burden of malaria in pregnancy. Lancet Infect Dis 2007;7:93-104.

52. WHO. WHO Evidence Review Group: Intermittent Preventive Treatment of malaria in pregnancy (IPTp) with SulfadoxinePyrimethamine (SP), 2012. 